\title{
Decentralised sliding mode control for a class of nonlinear interconnected systems
}

\author{
Jianqiu Mu, Xing-Gang Yan and Sarah K. Spurgeon
}

\begin{abstract}
In this paper, a decentralised control strategy based on sliding mode techniques is proposed for a class of nonlinear interconnected systems. Both matched uncertainties in the isolated subsystems and mismatched uncertainties associated with the interconnections are considered. Under mild conditions, sliding mode controllers for each subsystem are designed in a decentralised manner by only employing local information. Conditions are determined which enable information on the interconnections to be employed in decentralised controller design to reduce conservatism. The developed results are applied to an automated highway system. Simulation results pertaining to a high-speed following system are presented to demonstrate the effectiveness of the approach.
\end{abstract}

\section{INTRODUCTION}

For nonlinear interconnected systems, it is well known that uncertainties or modelling errors may seriously affect control system performance. Specifically, for large scale interconnected systems, uncertainties experienced by one subsystem not only affect its own performance but usually affect the other subsystems' performance as well due to the interactions among subsystems. Sliding mode control has been recognised as a powerful approach in dealing with uncertainties. When in the sliding mode, a closed loop system is completely insensitive to matched uncertainties[3], [4]. The sliding mode approach can also be used to deal with the systems in the presence of unmatched uncertainty [5] although the property of total insensitivity is frequently lost. However, in contrast to the case of centralised control, decentralised control can only use local information and thus the uncertainties within the interconnections may not be rejected, even if they are matched. Designing a decentralised control scheme to reject the effect of uncertainties in the interconnection terms is challenging.

The problem of robust decentralised controller design has received much attention and many results have been obtained. In [6], [7], [8], [9], only matched uncertainties are considered and bounds on the matched uncertainties are assumed to be linear or polynomial. In terms of mismatched uncertainties, in order to achieve asymptotic stability, some limitations are unavoidable. Mismatched uncertainties have been considered in [5], [10] where centralised dynamical feedback controllers are designed which need more resources to exchange information between subsystems. A class of constraints called integral quadratic constraints is imposed on the considered systems to limit the structure of the original

\footnotetext{
${ }^{1}$ Jianqiu Mu, Xing-Gang Yan and Sarah K. Spurgeon are with Instrumentation, Control and Embedded Systems Research Group, School of Engineering \& Digital Arts, University of Kent, CT2 7NT Canterbury, United Kingdom
}

systems [10]. In some cases, adaptive techniques are applied to estimate an upper bound on the mismatched uncertainty to counteract the effects [11]. This approach may be powerful when the uncertainty satisfies a linear growth condition. In [12], although the uncertainties are assumed to be functions, the system needs to be transformed into a special triangular structure. All the literature which considers mismatched uncertainties mentioned above inevitably requires extra resources and increases the system complexity. This may be unattractive from the viewpoint of implementation.

In this paper, a decentralised control strategy for a class of nonlinear interconnected systems is proposed based on a sliding mode control paradigm. In terms of the robustness, both matched and unknown interconnections with mismatched uncertainties are considered. Moreover, the uncertainties are assumed to be bounded by known functions which are employed within the control design to counteract the effects of the uncertainties. The bounds on the uncertainties take more general forms when compared with existing work. Based on the approach proposed in [3], a sliding surface for each subsystem is designed which together constitute a composite sliding surface for the interconnected system. A set of sufficient conditions is developed such that the corresponding sliding motion is asymptotically stable when the system is restricted to the designed sliding surface. Then, a decentralised sliding mode control is designed to drive the large-scale interconnected system to the sliding surface in finite time. It is shown that if the uncertainties/interconnections possess a superposition property, a decentralised control scheme may be designed to counteract the effect of the uncertainty. Finally, the developed decentralised control scheme is applied to an automated highway system. Simulation results relating to a high-speed car following system shows that the obtained results are effective.

\section{SYSTEM DESCRIPTION}

Consider a nonlinear large-scale interconnected system composed of $N$ subsystems where the $i$-th subsystem is described by

$$
\begin{aligned}
\dot{x}_{i}= & A_{i} x_{i}+B_{i}\left(u_{i}+\phi_{i}\left(t, x_{i}\right)\right)+\sum_{j=1}^{N} \Xi_{i j}\left(t, x_{j}\right) \\
& +\psi_{i}(t, x), \quad i=1,2, \ldots, N
\end{aligned}
$$

where $x_{i} \in \mathcal{D}_{i} \subset \mathcal{R}^{n_{i}}, u_{i} \in \mathcal{R}^{m_{i}}$ denote the state variables and inputs of the $i$-th subsystem, respectively. The matrix pairs $\left(A_{i}, B_{i}\right)$ are constant with appropriate dimensions. The matched uncertainties are denoted by $\phi_{i}\left(t, x_{i}\right)$. The term 
$\sum_{j=1}^{n} \Xi_{i j}\left(t, x_{j}\right)$ describes the known interconnection of the $i$-th subsystem. The nonlinear functions $\psi_{i}(t, x)$ represent the uncertain interconnections where $x=\operatorname{col}\left(x_{1}, x_{2}, \ldots, x_{n}\right)$. It is assumed that all the nonlinear functions are sufficiently smooth such that the unforced system has a unique continuous solution.

It should be noted that

$$
\sum_{j=1}^{N} \Xi_{i j}\left(t, x_{j}\right)=\Xi_{i i}\left(t, x_{i}\right)+\sum_{\substack{j \neq i \\ j=1}}^{N} \Xi_{i j}\left(t, x_{j}\right)
$$

In this case, $\Xi_{i i}\left(t, x_{i}\right)$ can be considered as the known nonlinearity in the $i$ th subsystem and the term $\sum_{\substack{j \neq i \\ j=1}}^{N} \Xi_{i j}\left(t, x_{j}\right)$ as the known interconnection within the $i$ th subsystem.

Assumption 1. The matrix pairs $\left(A_{i}, B_{i}\right)$ are controllable and $\operatorname{rank}\left(B_{i}\right)=m_{i}$ for $i=1,2, \ldots, N$.

Under the condition that $\operatorname{rank}\left(B_{i}\right)=m_{i}$ in Assumption 1, there exists an invertible matrix $\tilde{T}_{i} \in \mathcal{R}^{\left(n_{i} \times n_{i}\right)}$ such that after the following coordinate transformation $\tilde{x}_{i}=\tilde{T}_{i} x_{i}$, the matrix pairs $\left(A_{i}, B_{i}\right)$ with respect to the new coordinates $\tilde{x}_{i}$ have the following structure

$$
\begin{aligned}
\tilde{A}_{i} & =\left[\begin{array}{cc}
\tilde{A}_{i 1} & \tilde{A}_{i 2} \\
\tilde{A}_{i 3} & \tilde{A}_{i 4}
\end{array}\right]=\tilde{T}_{i} A_{i} \tilde{T}_{i}^{-1} \\
\tilde{B}_{i} & =\left[\begin{array}{c}
0 \\
\tilde{B}_{i 2}
\end{array}\right]=\tilde{T}_{i} B_{i}
\end{aligned}
$$

where $\tilde{A}_{i 1} \in \mathcal{R}^{\left(n_{i}-m_{i}\right) \times\left(n_{i}-m_{i}\right)}$ and the matrix $\tilde{B}_{i 2} \in$ $\mathcal{R}^{m_{i} \times m_{i}}$ is nonsingular for $i=1,2, \ldots, N$. It should be noted that the matrix $\tilde{T}_{i}$ can be obtained using basic matrix theory.

Assume that $\left(A_{i}, B_{i}\right)$ is controllable. From [3], it follows that the matrix pair $\left(\tilde{A}_{i 1}, \tilde{A}_{i 2}\right)$ in (3) is controllable. Then, there exists a matrix $K_{i} \in \mathcal{R}^{\left(n_{i}-m_{i}\right) \times m_{i}}$ such that $\tilde{A}_{i 1}-K_{i} \tilde{A}_{i 2}$ is Hurwitz stable. Considering the system (1), introduce a new transformation matrix as follows:

$$
T_{i}=\left[\begin{array}{cc}
I_{n_{i}-m_{i}} & 0 \\
K_{i} & I_{m_{i}}
\end{array}\right] \tilde{T}_{i}
$$

It is clear that the matrix $T_{i}$ is nonsingular. Define $z=$ $\operatorname{col}\left(z_{1}, z_{2}, \ldots, z_{N}\right)$ where $z_{i}=T_{i} x_{i}$. Then in this new coordinate system, system (1) has the following form

$$
\begin{aligned}
\dot{z}_{i}= & {\left[\begin{array}{cc}
A_{i 1} & A_{i 2} \\
A_{i 3} & A_{i 4}
\end{array}\right] z_{i}+\left[\begin{array}{c}
0 \\
\tilde{B}_{i 2}
\end{array}\right]\left(u_{i}+g_{i}\left(t, z_{i}\right)\right) } \\
& +\sum_{j=1}^{N} \Gamma_{i j}\left(t, z_{j}\right)+\delta_{i}(t, z)
\end{aligned}
$$

where $z_{i} \in T_{i}\left(D_{i}\right):=\Omega_{i}, A_{i 1}=\tilde{A}_{i 1}-\tilde{A}_{i 2} K_{i}$ is stable with $K_{i}$ given in (5), $T^{-1} \equiv: \operatorname{diag}\left\{T_{1}^{-1}, T_{2}^{-1}, \ldots, T_{N}^{-1}\right\}$ and for $i, j=1,2, \ldots, N$

$$
\begin{aligned}
g_{i}\left(t, z_{i}\right) & =\phi_{i}\left(t, T_{i}^{-1} z_{i}\right) \\
\Gamma_{i j}\left(t, z_{j}\right) & \triangleq\left[\begin{array}{c}
\Gamma_{i j}^{a}\left(t, z_{j}\right) \\
\Gamma_{i j}^{b}\left(t, z_{j}\right)
\end{array}\right]=T_{i} \Xi_{i j}\left(t, T_{j}^{-1} z_{j}\right) \\
\delta_{i}(t, z) & \triangleq\left[\begin{array}{c}
\delta_{i}^{a}(t, z) \\
\delta_{i}^{b}(t, z)
\end{array}\right]=T_{i} \psi_{i}\left(t, T^{-1} z\right)
\end{aligned}
$$

where

$$
\begin{array}{lll}
\Gamma_{i j}^{a}\left(t, z_{j}\right), & \delta_{i}^{a}(t, z) & \in \mathcal{R}^{\left(n_{i}-m_{i}\right)} \\
\Gamma_{i j}^{b}\left(t, z_{j}\right), & \delta_{i}^{b}(t, z) & \in \mathcal{R}^{m_{i}}
\end{array}
$$

For further analysis, now partition $z_{i}=\operatorname{col}\left(z_{i}^{a}, z_{i}^{b}\right)$ where $z_{i}^{a} \in \mathcal{R}^{n_{i}-m_{i}}$ and $z_{i}^{b} \in \mathcal{R}^{m_{i}}$. Then the system (6) can be rewritten in the following form

$$
\begin{aligned}
\dot{z}_{i}^{a}= & A_{i 1} z_{i}^{a}+A_{i 2} z_{i}^{b}+\sum_{j=1}^{N} \Gamma_{i j}^{a}\left(t, z_{j}\right)+\delta_{i}^{a}(t, z) \\
\dot{z}_{i}^{b}= & A_{i 3} z_{i}^{a}+A_{i 4} z_{i}^{b}+\tilde{B}_{i 2}\left(u_{i}+g_{i}\left(t, z_{i}\right)\right) \\
& +\sum_{j=1}^{N} \Gamma_{i j}^{b}\left(t, z_{j}\right)+\delta_{i}^{b}(t, z)
\end{aligned}
$$

where the matrix $A_{i 1}$ in (10) is stable.

Assumption 2. There exist known continuous functions $\rho_{i}\left(t, z_{i}\right), \eta_{i}^{a}(t, z)$ and $\eta_{i}^{b}(t, z)$ such that for $i, j=1,2, \ldots, N$

(i) $\left\|g_{i}\left(t, z_{i}\right)\right\| \leq \rho_{i}\left(t, z_{i}\right)$

(ii) $\left\|\delta_{i}^{a}(t, z)\right\| \leq \eta_{i}^{a}(t, z)\|z\|$

(iii) $\left\|\delta_{i}^{b}(t, z)\right\| \leq \eta_{i}^{b}(t, z)$

\section{Stability Analysis of the Sliding Mode}

Choose the local sliding surface for the $i$ th subsystem of the large-scale interconnected system (6) as follows:

$$
\sigma_{i}\left(z_{i}\right) \equiv: z_{i}^{b}=0, \quad i=1,2, \ldots, N .
$$

Then, the composite sliding surface for the interconnected system (6) is chosen as

$$
\sigma(z)=0
$$

where $\sigma(z) \equiv: \operatorname{col}\left(z_{1}^{b}, z_{2}^{b}, \ldots, z_{N}^{b}\right)$

Since $A_{i 1}$ in (10) is stable, for any $Q_{i}>0$, the following Lyapunov equation has a unique solution $P_{i}>0$ such that

$$
A_{i 1}^{\tau} P_{i}+P_{i} A_{i 1}=-Q_{i}, \quad i=1,2, \ldots, N .
$$

During sliding motion, $z_{i}^{b}=0$ for $i=1,2, \ldots, N$. Then, the sliding mode dynamics for the system (10)-(11) associated with the designed sliding surface (13) can be described by

$$
\dot{z}_{i}^{a}=A_{i 1} z_{i}^{a}+\sum_{j=1}^{n} \Gamma_{i j}^{s}\left(t, z_{j}^{a}\right)+\delta_{i}^{s}\left(t, z_{1}^{a}, z_{2}^{a}, \ldots, z_{N}^{a}\right)
$$

where

$$
\begin{aligned}
\Gamma_{i j}^{s}\left(t, z_{j}^{a}\right) & :=\left.\Gamma_{i j}^{a}\left(t, z_{j}\right)\right|_{z_{j}^{b}=0} \\
\delta_{i}^{s}\left(t, z_{1}^{a}, z_{2}^{a}, \ldots, z_{N}^{a}\right) & :=\left.\delta_{i}^{a}(t, z)\right|_{\left(z_{1}^{b}, z_{2}^{b}, \ldots, z_{N}^{b}\right)=0}
\end{aligned}
$$

Here $\Gamma_{i j}^{a}\left(t, z_{j}\right)$ and $\delta_{i}^{a}(t, z)$ are defined in (8) and (9) respectively.

Assumption 3. The function $\Gamma_{i j}^{s}(\cdot)$ has the following decomposition:

$$
\Gamma_{i j}^{s}\left(t, z_{j}^{a}\right)=\tilde{\Gamma}_{i j}^{s}\left(t, z_{j}^{a}\right) z_{j}^{a}
$$


where $\tilde{\Gamma}_{i j}^{s}\left(t, z_{j}^{a}\right)$ is an appropriately-dimensioned matrix function for $i, j=1,2, \ldots, N$.

Under Assumptions 1-3, a reduced order interconnected system composed of $N$ subsystems with dimension $n_{i}-m_{i}$ is obtained as follows

$$
\dot{z}_{j}^{a}=A_{i 1} z_{j}^{a}+\sum_{j=1}^{n} \tilde{\Gamma}_{i j}^{s}\left(t, z_{j}^{a}\right) z_{j}^{a}+\delta_{i}^{s}\left(t, z_{1}^{a}, z_{2}^{a}, \ldots, z_{N}^{a}\right)
$$

which represents the sliding mode dynamics relating to the sliding surface (13), where $z_{i}^{a} \in \mathcal{R}^{n_{i}-m_{i}}$ and $\tilde{\Gamma}_{i j}^{s}\left(t, z_{j}^{a}\right)$ is defined in (18).

Lemma 1: For terms $\delta_{i}^{s}\left(t, z_{1}^{a}, z_{2}^{a}, \ldots, z_{N}^{a}\right)$ in system (19), if condition (ii) in Assumption 2 holds, then there exist continuous functions $\gamma_{i j}(\cdot)$ such that

$$
\left\|\delta_{i}^{s}\left(t, z_{1}^{a}, z_{2}^{a}, \ldots, z_{N}^{a}\right)\right\| \leq \sum_{j=1}^{N} \gamma_{i j}\left(t, z^{a}\right)\left\|z_{j}^{a}\right\|
$$

where $z^{a}=\operatorname{col}\left(z_{1}^{a}, z_{2}^{a}, \ldots, z_{N}^{a}\right)$.

Proof. From the definition of $\delta_{i}^{s}(\cdot)$ in (17), it follows that

$$
\delta_{i}^{s}\left(t, z_{1}^{a}, z_{2}^{a}, \ldots, z_{N}^{a}\right)=\delta_{i}^{a}\left(t, z_{1}^{a}, 0, z_{2}^{a}, 0, \ldots, z_{N}^{a}, 0\right)
$$

From condition (ii) in Assumption 2,

$$
\left\|\delta_{i}^{a}(t, z)\right\| \leq \eta_{i}^{a}(t, z)\|z\|
$$

From (21) and (22), it follows that

$$
\begin{aligned}
& \left\|\delta_{i}^{s}\left(t, z_{1}^{a}, z_{2}^{a}, \ldots, z_{N}^{a}\right)\right\| \\
= & \left\|\delta_{i}^{a}\left(t, z_{1}^{a}, 0, z_{2}^{a}, 0, \ldots, z_{N}^{a}, 0\right)\right\| \\
\leq & \eta_{i}^{a}\left(t, z_{1}^{a}, 0, z_{2}^{a}, 0, \ldots, z_{N}^{a}, 0\right)\left\|z^{a}\right\| \\
\leq & \sum_{j=1}^{N} \eta_{i}^{a}\left(t, z_{1}^{a}, 0, z_{2}^{a}, 0, \ldots, z_{N}^{a}, 0\right)\left\|z_{j}^{a}\right\| \\
\leq & \sum_{j=1}^{N} \gamma_{i j}\left(t, z_{j}^{a}\right)\left\|z_{j}^{a}\right\|
\end{aligned}
$$

where $\gamma_{i j}\left(t, z_{j}^{a}\right)=\eta_{i}^{a}\left(t, z_{1}^{a}, 0, z_{2}^{a}, 0, \ldots, z_{N}^{a}, 0\right)$ for $i=$ $1,2, \ldots, N$. Hence the result follows.

Theorem 1: Consider the sliding mode dynamics given in equation (19). Under Assumptions 1-3, the sliding motion governed by (19) is asymptotically stable if there exists a domain $\Omega_{z^{a}}$ of the origin in $z^{a} \in \mathcal{R}^{\sum_{i=1}^{N}\left(n_{i}-m_{i}\right)}$ such that

$$
M^{\tau}+M>0
$$

in $\Omega_{z^{a}} \backslash\{0\}$ where $M=\left(m_{i j}\right)_{N \times N}$ and for $i, j=$ $1,2, \ldots, N$

$m_{i j}=\left\{\begin{array}{cc}\lambda_{\min }\left(Q_{i}\right)-2\left\|P_{i}\right\| \gamma_{i i}\left(t, z_{i}^{a}\right)-\varsigma_{i i}\left(t, z_{i}^{a}\right), & i=j \\ -\varsigma_{i j}\left(t, z_{j}^{a}\right)-2\left\|P_{i}\right\| \gamma_{i j}\left(t, z_{j}^{a}\right), & i \neq j\end{array}\right.$

where $P_{i}$ and $Q_{i}$ satisfy (14), and the functions $\varsigma_{i j}(\cdot)$ are defined by

$$
\varsigma_{i j}\left(t, z_{j}^{a}\right): \equiv\left\|P_{i} \tilde{\Gamma}_{i j}^{s}\left(t, z_{j}^{a}\right)+\left(\tilde{\Gamma}_{i j}^{s}\right)^{\tau}\left(t, z_{j}^{a}\right) P_{i}\right\|
$$

with $\tilde{\Gamma}_{i j}^{s}\left(t, z_{j}^{a}\right)$ given by (18), and $\gamma_{i j}\left(t, z_{j}^{a}\right)$ determined by (20).
Proof. For system (19), consider the Lyapunov function candidate

$$
V\left(t, z_{1}^{a}, z_{2}^{a}, \ldots, z_{N}^{a}\right)=\sum_{i=1}^{N}\left(z_{j}^{a}\right)^{\tau} P_{i} z_{j}^{a}
$$

where $P_{i}$ satisfies equation (14)

Then, the time derivative of $V\left(t, z_{1}^{a}, z_{2}^{a}, \ldots, z_{N}^{a}\right)$ along the trajectories of system (19) is given by

$$
\begin{aligned}
\dot{V}= & \sum_{i=1}^{N}\left\{-\left(z_{j}^{a}\right)^{\tau} Q_{i} z_{i}^{a}+2\left(z_{j}^{a}\right)^{\tau} P_{i} \delta_{i}^{s}\left(t, z_{1}^{a}, z_{2}^{a}, \ldots, z_{N}^{a}\right)\right. \\
& +\sum_{j=1}^{n}\left(z_{j}^{a}\right)^{\tau}\left(P_{i} \tilde{\Gamma}_{i j}^{s}\left(t, z_{j}^{a}\right)\right. \\
& \left.\left.+\left(\tilde{\Gamma}_{i j}^{s}\right)^{\tau}\left(t, z_{j}^{a}\right) P_{i}\right) z_{j}^{a}\right\}
\end{aligned}
$$

where (14) is used above. From (20), it follows that

$$
\begin{aligned}
\dot{V} \leq & \sum_{i=1}^{N}\left\{-\lambda_{\min }\left(Q_{i}\right)\left\|z_{i}^{a}\right\|^{2}\right. \\
& +2\left\|z_{i}^{a}\right\|\left\|P_{i}\right\|\left\|\delta_{i}^{s}\left(t, z_{1}^{a}, z_{2}^{a}, \ldots, z_{N}^{a}\right)\right\|+ \\
& \left.\sum_{j=1}^{N}\left\|P_{i} \tilde{\Gamma}_{i 1}^{s}\left(t, z_{j}^{a}\right)+\left(\tilde{\Gamma}_{i j}^{s}\left(t, z_{j}^{a}\right)\right)^{\tau} z_{j}^{a} P_{i}\right\|\left\|z_{i}^{a}\right\|\left\|z_{j}^{a}\right\|\right\} \\
\leq & \sum_{i=1}^{N}\left\{-\lambda_{\min }\left(Q_{i}\right)\left\|z_{i}^{a}\right\|^{2}+\sum_{j=1}^{N} \varsigma_{i j}\left(t, z_{j}^{a}\right)\left\|z_{i}^{a}\right\|\left\|z_{j}^{a}\right\|\right. \\
& \left.+2\left\|z_{i}^{a}\right\|\left\|P_{i}\right\| \sum_{j=1}^{N} \gamma_{i j}\left(t, z_{j}^{a}\right)\left\|z_{j}^{a}\right\|\right\} \\
= & -\sum_{i=1}^{N}\left\{\lambda_{\min }\left(Q_{i}\right)-2\left\|P_{i}\right\| \gamma_{i i}\left(t, z_{i}^{a}\right)\right. \\
& \left.-\varsigma_{i i}\left(t, z_{i}^{a}\right)\right\}\left\|z_{i}^{a}\right\|^{2}+ \\
& \sum_{i=1}^{N} \sum_{\substack{j=1 \\
j \neq i}}^{N}\left\{\varsigma_{i j}\left(t, z_{j}^{a}\right)+2\left\|P_{i}\right\| \gamma_{i j}\left(t, z_{j}^{a}\right)\right\}\left\|z_{i}^{a}\right\|\left\|z_{j}^{a}\right\| \\
= & -\frac{1}{2} Y^{\tau}\left(M^{\tau}+M\right) Y \leq 0
\end{aligned}
$$

where $Y \equiv: \operatorname{col}\left(\left\|z_{1}^{a}\right\|, \ldots,\left\|z_{N}^{a}\right\|\right)$.

Thus, the conclusion follows from $M^{\tau}+M>0$.

\section{Decentralised Sliding MOde CONTROL DESIGN}

For the nonlinear interconnected system (1), the corresponding condition is described by

$$
\sum_{i=1}^{N} \frac{\sigma_{i}^{\tau}\left(z_{i}\right) \dot{\sigma}_{i}\left(z_{i}\right)}{\left\|\sigma_{i}\left(z_{i}\right)\right\|}<0
$$

where $\sigma_{i}\left(z_{i}\right)$ is defined by (13). It should be noted that the condition (27) is proposed in [14] and has been widely used [13].

Consider the expression

$$
\eta_{i}^{b}(t, z)=\sum_{j=1}^{N} \mu_{i j}\left(t, z_{j}\right)+\nu_{i}(t, z)
$$


where $\nu_{i}(t, z)$ represents all the coupling terms which cannot be included in the term $\sum_{j=1}^{N} \mu_{i j}\left(t, z_{j}\right)$

For $i=1,2, \ldots, N$, the following control scheme is proposed:

$$
\begin{aligned}
u_{i}= & -\tilde{B}_{i 2}^{-1}\left\{A_{i 3} z_{i}^{a}+A_{i 4} z_{i}^{b}+\sum_{j=1}^{N} \Gamma_{j i}^{b}\left(t, T_{i}^{-1} z_{i}\right)\right\} \\
& -\tilde{B}_{i 2}^{-1} \operatorname{sgn}\left(z_{i}^{b}\right)\left\{\left\|\tilde{B}_{i 2}\right\| \rho_{i}\left(t, z_{i}\right)\right. \\
& \left.+\sum_{j=1}^{N} \mu_{j i}\left(t, z_{i}\right)+\zeta_{i}\left(t, z_{i}\right)\right\}
\end{aligned}
$$

where the $\rho_{i}\left(t, z_{i}\right)$ are defined in Assumption 2, $\mu_{j i}\left(t, z_{i}\right)$ satisfy (28) and $\zeta_{i}\left(t, z_{i}\right)$ is a reachability function which will be defined later.

Theorem 2: Consider the nonlinear interconnected system (6). Under Assumptions 1-3, the decentralised control (29) is able to drive the system (1) to the composite sliding surface (13) and maintains a sliding motion on it thereafter if in the considered domain $\Omega=\Omega_{1} \times \Omega_{2} \cdots \times \Omega$, the functions $\zeta_{i}\left(t, z_{i}\right)$ in (29) satisfy

$$
\sum_{i=1}^{N} \zeta_{i}\left(t, z_{i}\right)>\sum_{i=1}^{N} \nu_{i}(t, z)
$$

in $\Omega \backslash\{0\}$ for all $t>0$ with $\nu_{i}(t, z)$ defined in (28).

Proof. From (13), for $i=1,2, \ldots, N$

$$
\begin{aligned}
\dot{\sigma}_{i}\left(z_{i}\right)=\dot{z}_{i}^{b}= & A_{i 3} z_{i}^{a}+A_{i 4} z_{i}^{b} \\
& +\tilde{B}_{i 2}\left(u_{i}+\phi_{i}\left(t, T_{i}^{-1} z_{i}\right)\right) \\
& +\sum_{j=1}^{N} \Gamma_{i j}^{b}\left(t, z_{j}\right)+\delta_{i}^{b}(t, z)
\end{aligned}
$$

Substituting (29) into (31),

$$
\begin{aligned}
& \sum_{i=1}^{N} \frac{\sigma_{i}^{\tau}\left(z_{i}\right) \dot{\sigma}_{i}\left(z_{i}\right)}{\left\|\sigma_{i}\left(z_{i}\right)\right\|} \\
= & \sum_{i=1}^{N}\left\{\frac{\left(z_{i}^{b}\right)^{\tau}}{\left\|z_{i}^{b}\right\|}\left\{\delta_{i}^{b}(t, z)+\tilde{B}_{i 2} \phi_{i}\left(t, T_{i}^{-1} z_{i}\right)\right\}\right. \\
& \left.-\left\|\tilde{B}_{i 2}\right\| \rho_{i}\left(t, z_{i}\right)-\sum_{j=1}^{N} \mu_{j i}\left(t, z_{i}\right)-\zeta_{i}\left(t, z_{i}\right)\right\} \\
& +\frac{\left(z_{i}^{b}\right)^{\tau}}{\left\|z_{i}^{b}\right\|}\left\{\sum_{i=1}^{N} \sum_{j=1}^{N} \Gamma_{i j}^{b}\left(t, z_{j}\right)-\sum_{i=1}^{N} \sum_{j=1}^{N} \Gamma_{j i}^{b}\left(t, z_{i}\right)\right\} \\
\leq & \sum_{i=1}^{N}\left\|\tilde{B}_{i 2} \phi_{i}\left(t, T_{i}^{-1} z_{i}\right)\right\|+\sum_{i=1}^{N}\left\|\delta_{i}^{b}(t, z)\right\| \\
& -\sum_{i=1}^{N}\left\|\tilde{B}_{i 2}\right\| \rho_{i}\left(t, z_{i}\right)-\sum_{i=1}^{N} \sum_{j=1}^{N} \mu_{j i}\left(t, z_{i}\right) \\
& -\sum_{i=1}^{N} \zeta_{i}\left(t, z_{i}\right)
\end{aligned}
$$

From Assumption 3,

$$
\begin{aligned}
& \sum_{i=1}^{N}\left\|\delta_{i}^{b}\left(t, T^{-1} z\right)\right\| \\
\leq & \sum_{i=1}^{N} \sum_{j=1}^{N} \mu_{i j}\left(t, z_{j}\right)+\sum_{i=1}^{N} \nu_{i}(t, z) \\
= & \sum_{i=1}^{N} \sum_{j=1}^{N} \mu_{j i}\left(t, z_{i}\right)+\sum_{i=1}^{N} \nu_{i}(t, z)
\end{aligned}
$$

and

$$
\begin{aligned}
\left\|\tilde{B}_{i 2} \phi_{i}\left(t, T_{i}^{-1} z_{i}\right)\right\| & \leq\left\|\tilde{B}_{i 2}\right\|\left\|\phi_{i}\left(t, T_{i}^{-1} z_{i}\right)\right\| \\
& \leq\left\|\tilde{B}_{i 2}\right\| \rho_{i}\left(t, z_{i}\right)
\end{aligned}
$$

Substituting inequalities (33) and (34) into (32)

$$
\sum_{i=1}^{N} \frac{\sigma_{i}^{\tau} \dot{\sigma}_{i}}{\left\|\sigma_{i}\right\|} \leq-\sum_{i=1}^{N} \zeta_{i}\left(t, z_{i}\right)+\sum_{i=1}^{N} \nu_{i}(t, z)<0
$$

Then the reachability condition (27) is satisfied. Hence, the result follows.

\section{Simulation Example}

In order to achieve high traffic flow rates and reduce congestion, an automated highway system has been developed [15]. During the automated driving process, vehicles are driven automatically with both on board lateral and longitudinal controllers. The lateral controller is used to steer the vehicle and the longitudinal controller is used to follow a lead vehicle at a safe distance. The stability and the robustness of the car-following system will be considered as case study to demonstrate the theoretical results. The dynamics of the car-following system is described by [1]

$$
\begin{aligned}
\dot{\xi}_{i} & =v_{i}-v_{(i-1)} \\
\dot{v}_{i} & =\frac{1}{m_{i}}\left(-A_{i p} v_{i}^{2}-d_{i}+f_{i}\right) \\
\dot{f}_{i} & =\frac{1}{\kappa_{i}}\left(-f_{i}+u_{i}\right)
\end{aligned}
$$

where $\xi_{i}$ represents the distance between the $i$ th and the $(i-1)$ th vehicle, $v_{i}$ is the velocity of the $i$ th car and $f_{i}$ is the force applied to the longitudinal dynamics of the $i$ th vehicle, where if $f_{i}>0$ a forward driving force occurs and if $f_{i}<0$, then a braking force takes place. $m_{i}$ is the mass of the $i$ th car, $d_{i}$ and $\kappa_{i}$ are the constant frictional force and the engine brake time constant. The signal $u_{i}$ is the control variable, where if $u_{i}>0$, a throttle input results, and if $u_{i}<0$ then a braking input occurs. Choosing the same parameters as those in [1]:

$$
\begin{array}{ll}
m_{i}=1300 \mathrm{~kg}, & A_{i p}=0.3 \mathrm{Ns}^{2} / \mathrm{m}^{2}, \quad d_{i}=100 \mathrm{~N} \\
\kappa_{i}=0.2 \mathrm{~s}, & v_{0}=20 \mathrm{~m} / \mathrm{s}
\end{array}
$$

Define $\xi_{d}=10$ and $v_{d}=v_{0}$ as the ideal distance between every adjacent car and an ideal driving velocity, respectively. 
Let

$$
\begin{aligned}
& x_{i 1}=l_{i}-l_{d} \\
& x_{i 2}=v_{i}-v_{d} \\
& x_{i 3}=\frac{f_{i}-A_{i p} v_{0}^{2}-d_{i}}{1000}
\end{aligned}
$$

for $i=1,2,3$, and consider the system in the domain

$$
D_{i}=\left\{x_{i} \in \mathcal{R}^{3}\left|x_{i 1} \geq-\xi_{d},\right| x_{i 2}|\leq 20,| x_{i 3} \mid \leq 20\right\}
$$

Then, the 3-car following system can be described in the form of (1) by the following equations

$$
\begin{aligned}
\dot{x}_{i}= & \underbrace{\left[\begin{array}{ccc}
0 & 1 & 0 \\
0 & \frac{-12}{1300} & \frac{10}{13} \\
0 & 0 & -5
\end{array}\right]}_{A_{i}} x_{i} \\
& +\underbrace{\left[\begin{array}{c}
0 \\
0 \\
0.005
\end{array}\right]}_{B}\left(u_{i}+220+\phi_{i}\left(x_{i}, t\right)\right) \\
& +\underbrace{\left[\begin{array}{c}
-x_{(i-1) 2} \\
0 \\
0
\end{array}\right]}_{\Xi_{i(i-1)}}+\underbrace{\left[\begin{array}{c}
0 \\
-\frac{0.3}{1300} x_{i 2}^{2} \\
0
\end{array}\right]}_{\Xi_{i i}} \\
& +\psi_{i}(t, x),
\end{aligned}
$$

where $\Xi_{i j}=0$ if $i \neq j$ and $j \neq i-1$, and

$$
\Xi_{i 0}=\left[\begin{array}{c}
-x_{02} \\
0 \\
0
\end{array}\right]=\left[\begin{array}{c}
-v_{0}+v_{d} \\
0 \\
0
\end{array}\right]=0
$$

The unknown matched uncertainty $\phi_{i}\left(x_{i}, t\right)$ is assumed to satisfy

$$
\begin{aligned}
\left\|\phi_{1}\left(x_{1}, t\right)\right\| & \leq 20\left|x_{11}+x_{12}\right|+80\left|x_{13}\right| \\
\left\|\phi_{2}\left(x_{2}, t\right)\right\| & \leq 50\left|x_{21}+x_{22}\right|+31\left|x_{23}\right|
\end{aligned}
$$

By using the algorithm in [3], the coordinate transformation $z_{i}=T_{i} x_{i}$ for $i=1,2,3$ can be obtained with $T_{i}$ defined by

$$
\begin{aligned}
& T_{1}=T_{2}=\left[\begin{array}{ccc}
1 & 0 & 0 \\
0 & 1 & 0 \\
3.9 & 3.9 & 1
\end{array}\right] \\
& T_{3}=\left[\begin{array}{ccc}
1 & 0 & 0 \\
0 & 1 & 0 \\
3.9 & 2.6 & 1
\end{array}\right]
\end{aligned}
$$

Then the system (42) is transformed into the form (10)(11) with

$$
\left[\begin{array}{ll}
A_{i 1} & A_{i 2} \\
A_{i 3} & A_{i 4}
\end{array}\right]=\left[\begin{array}{cc:c}
0 & 1 & 0 \\
-3 & -3.0092 & 0.7692 \\
\hdashline 7.8 & 11.664 & -2
\end{array}\right]
$$

for $i=1,2$ and

$$
\begin{aligned}
{\left[\begin{array}{ll}
A_{31} & A_{32} \\
A_{33} & A_{34}
\end{array}\right] } & =\left[\begin{array}{cc:c}
0 & 1 & 0 \\
-3 & -2.0092 & 0.7692 \\
\hdashline 11.7 & 11.676 & -3
\end{array}\right] \\
B_{1} & =B_{2}=B_{3}=\left[\begin{array}{c}
0 \\
0 \\
\hdashline 0.005
\end{array}\right] \\
\Gamma_{i i}\left(t, z_{j}\right) & =\left[\begin{array}{l}
\Gamma_{i i}^{a}\left(t, z_{j}\right) \\
\Gamma_{i i}^{b}\left(t, z_{j}\right)
\end{array}\right]=\left[\begin{array}{c}
0 \\
-\frac{0.3}{1300} x_{i 2}^{2} \\
\hdashline-\frac{1.17}{1300} x_{i 2}^{2}
\end{array}\right]
\end{aligned}
$$

for $i=1,2$ and

$$
\begin{aligned}
\Gamma_{33}\left(t, z_{3}\right) & =\left[\begin{array}{c}
\Gamma_{33}^{a}\left(t, z_{3}\right) \\
\Gamma_{33}^{b}\left(t, z_{3}\right)
\end{array}\right]=\left[\begin{array}{c}
0 \\
-\frac{0.3}{1300} x_{32}^{2} \\
-\frac{0.78}{1300} x_{32}^{2}
\end{array}\right] \\
\Gamma_{i(i-1)} & =\left[\begin{array}{c}
\Gamma_{i(i-1)}^{a}\left(t, z_{j}\right) \\
\Gamma_{i(i-1)}^{b}\left(t, z_{j}\right)
\end{array}\right] \\
& =\left[\begin{array}{c}
-x_{(i-1) 2} \\
0 \\
-3.9 x_{(i-1) 2}
\end{array}\right], \quad i=2,3
\end{aligned}
$$

From (43) and (44) the unknown interconnections satisfy

$$
\begin{aligned}
& \delta_{1}^{a}(t, z) \leq 0.16 \cos ^{2}\left(z_{11}\right)\left\|z_{1}\right\| \\
& \delta_{1}^{b}(t, z) \leq \underbrace{0.8825 \cos ^{2}\left(z_{11}\right)\left\|z_{1}\right\|}_{\mu_{11}\left(t, z_{1}\right)}+\underbrace{0.7\left|z_{22}\right|}_{\mu_{12}\left(t, z_{2}\right)} \\
& \delta_{2}^{a}(t, z) \leq \underbrace{0.08 \sin ^{2}\left(z_{22}\right)\|z\|}_{\mu_{22}\left(t, z_{2}\right)} \\
& \delta_{2}^{b}(t, z) \leq \underbrace{0.4412 \sin ^{2}\left(z_{22}\right)\left\|z_{2}\right\|}_{\nu_{2}(t, z)}+ \\
& \delta_{3}^{b}(t, z) \leq \underbrace{0.6412 \sin ^{2}\left(z_{22}\right)\left\|z_{1}\right\|+0.4412 \sin ^{2}\left(z_{22}\right)\left\|z_{3}\right\|}_{\mu_{32}\left(t, z_{2}\right)}
\end{aligned}
$$

It is clear that the known nonlinear interconnections $\Gamma_{i j}\left(t, z_{j}\right)$ in equation (18) can be expressed as

$$
\begin{aligned}
\Gamma_{i i}^{s}= & {\left[\begin{array}{ccc}
0 & 0 & 0 \\
0 & -\frac{0.3}{1300} x_{i 2} & 0 \\
0 & -\frac{1.17}{1300} x_{i 2} & 0
\end{array}\right], \quad i=1,2 } \\
\Gamma_{33}^{s}= & {\left[\begin{array}{ccc}
0 & 0 & 0 \\
0 & -\frac{0.3}{1300} x_{32} & 0 \\
0 & -\frac{0.78}{1300} x_{32} & 0
\end{array}\right] } \\
\Gamma_{21}^{s}= & \Gamma_{32}^{s}=\left[\begin{array}{ccc}
0 & -1 & 0 \\
0 & 0 & 0 \\
0 & -3.9 & 0
\end{array}\right]
\end{aligned}
$$

which, by direct verification, satisfy (18). Now define the sliding surface as

$$
\sigma\left(z_{i}\right)=z_{i 3}, \quad i=1,2,3
$$


Then, when the sliding motion take place, from Lemma 1

$$
\begin{aligned}
\delta_{1}^{a}\left(t, z_{1}^{a}, z_{2}^{a}, z_{3}^{a}\right) & \leq 0.16 \cos ^{2}\left(z_{11}\right)\left\|z_{1}^{a}\right\| \\
\delta_{2}^{a}\left(t, z_{1}^{a}, z_{2}^{a}, z_{3}^{a}\right) & \leq \sum_{j=1}^{3} 0.08 \sin ^{2}\left(z_{22}\right)\left\|z_{j}^{a}\right\|
\end{aligned}
$$

Choosing $Q_{1}=8 I_{2}, Q_{2}=Q_{3}=I_{2}$ and solving the Lyapunov equation (14) yields

$$
\begin{aligned}
& P_{1}=\left[\begin{array}{cc}
5.7846 & -4 \\
-4 & 5.3170
\end{array}\right] \\
& P_{2}=\left[\begin{array}{cc}
0.7231 & -0.5 \\
-0.5 & 0.6646
\end{array}\right] \\
& P_{3}=\left[\begin{array}{cc}
0.6667 & -0.5 \\
-0.5 & 0.9954
\end{array}\right]
\end{aligned}
$$

Then, the matrix function $M$ of the entire system can be obtained. It is straightforward to verify that in the domain $\Omega$,

$$
M^{\tau}+M>0
$$

It follows from Theorem 1 that the designed sliding mode is asymptotically stable.

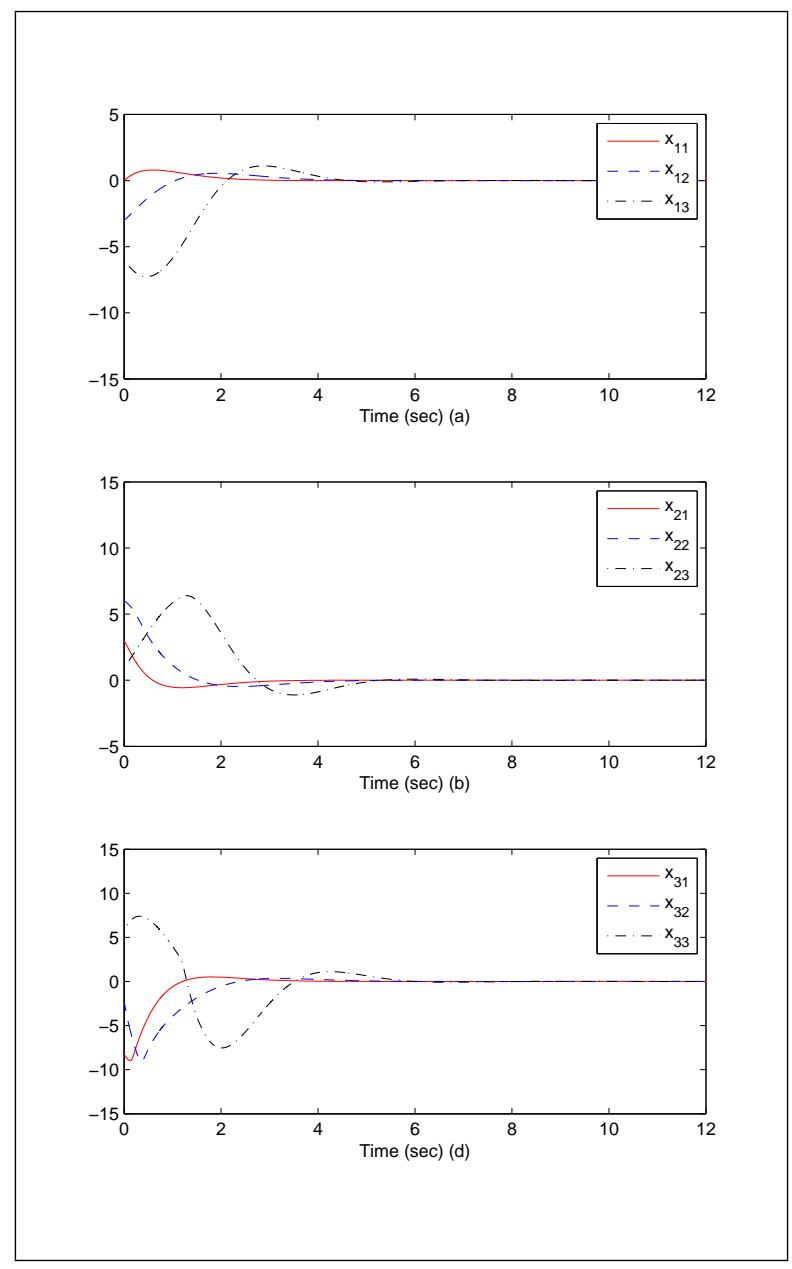

Fig. 1. The time responses of state variable of system (42)

From (29), the controller $u_{i}$ for $i=1,2,3$ where $\zeta_{1}=$ $20+0.4413\left\|z_{1}\right\|, \zeta_{2}=30, \zeta_{3}=20+0.4413\left\|z_{3}\right\|$ are well defined and the condition (30) in Theorem 2 is satisfied in the domain $\Omega$. The time responses of the system states is shown in Fig.1. The simulation results show that the proposed approach is effective.

\section{CONCLUSION}

A decentralised state feedback sliding mode control law has been proposed to stabilise a class of nonlinear interconnected systems with known and unknown interconnections asymptotically in the considered domain. Both matched and mismatched uncertainties are considered. The bounds on the uncertainty can be functions instead of constants or polynomial bounds as have been considered in previous work. Both known interconnections and the bounds on the unknown interconnections have been fully considered in the control design to reduce the conservatism. The developed results are applicable to a wide class of interconnected systems. Simulations based on a car-following systems have been presented to shows that the results obtained are effective.

\section{REFERENCES}

[1] J. T. Spooner, and K. M. Passino, Adaptive Control of a class of decentralised Nonlinear Systems, IEEE Trans. on Automat. Control, vol. 41, no. 2, pp. 280-284, 1996.

[2] X.-G. Yan, S. K. Spurgeon and C. Edwards, Decentralised output feedback sliding mode control of nonlinear large-scale systems with uncertainties. J. Optimization Theory and Appl., vol. 119, no. 3, pp. 597-614, 2003.

[3] C. Edwards and S. K. Spurgeon, Sliding mode control: Theory and applications. London: Taylor \& Francis, 1998.

[4] V. Utkin, J. Guldner and M. Shijun, Sliding Mode Control in Electromechanical Systems. London: Taylor \& Francis, 1998.

[5] X.-G. Yan, S. K. Spurgeon and C. Edwards, Dynamic sliding mode control for a class of systems with mismatched uncertainty, European J. Control, vol. 11, no. 1, pp. 1-10, 2005.

[6] M. Aldeen and H. Trinh, Decentralised feedback controllers for uncertain interconnected dynamic systems. IEE Proc. Part D: Control Theory Appl., vol. 140, no. 6, pp. 429-434, 1993.

[7] C.-F. Cheng, Disturbances attenuation for interconnected systems, Int. J. Control, vol. 66, no. 2, pp. 213-224, 1997.

[8] L. Jiang, Q. H. Wu and J. W. Wen, Decentralised nonlinear adaptive control for multimachine power systems via high-gain perturbation observer, IEEE Trans. on Circuits and Systems (I), vol. 51, no. 10, pp. 2052-2059, Oct. 2004

[9] M. Yang, F. Yang, C.-S. Wang, and P. Wang, Decentralised sliding mode load frequency control for multi-area power systems, IEEE Trans. on Power Systems, vol. 28, no. 4, pp. 4301-4309, 2013.

[10] V. A. Ugrinovskii, I. R. Petersen, A. V. Savkin and E. Y. Ugrinovskaya, Decentralised state-feedback stabilization and robust control of uncertain large-scale systems with intergrally constrained interconnection, Systems and Control Letters, vol. 41, no. 2, pp. 107-119, 2000.

[11] C. C. Cheng and Y. CHang, Design of decentralised adaptive sliding mode controllers for large-scale systems with mismatched perturbations, Int. J. Control, vol. 81, no. 10, pp. 1507-1518, 2008.

[12] Z.-P. Jiang, Recent developments in decentralised nonlinear control, in 2004 8th Int. Conf. on Control, Automation, Robotics and Vision, Kunming, China, 2004.

[13] X.-G. Yan, C. Edwards, S. K. Spurgeon, Decentralised robust sliding mode control for a class of nonlinear interconnceted systems by static output feedback, Automatica, vol. 40, pp. 613-620, 2004

[14] K. C. Hsu, Decentralised variable-structure control design for uncertain large-scale systems with series nonlinearities, Int. J. control, vol. 68, no. 6, pp. 1231-1240, 1997.

[15] S. E. Shladover, C. A. Desoer, J. K. Hedrick, M. Tomizuka, J. Walrand, W.-B. Zhang, D. H. McMahon, H. Peng, S. Sheikholeslam, N. McKeown, Automatic vehicle control developments in the PATH program, IEEE Trans. Veh. Technol., vol. 40, no. 1, pp. 114-130, 1991. 